$\mathrm{DFPD} / \mathrm{TH} / 95-39$

SISSA-AP/95-74

\title{
A stochastic approach to thermal fluctuations during a first order electroweak phase transition
}

\author{
Fabrizio Illuminati ${ }^{(a)(c)}$ 円 and Antonio Riotto ${ }^{(b)(c)} \mathrm{g}$ \\ (a) Dipartimento di Fisica, Università di Padova, via F. Marzolo 8, I-35131 Padova, Italy \\ (b) International School for Advanced Studies, SISSA, via Beirut 2, I-34014 Trieste, Italy \\ (c) Istituto Nazionale di Fisica Nucleare, Sezione di Padova, I-35131 Padova, Italy.
}

\begin{abstract}
We investigate the role played by subcritical bubbles at the onset of the electroweak phase transition. Treating the configuration modelling the thermal fluctuations around the homogeneous zero configuration of the Higgs field as a stochastic variable, we describe its dynamics by a phenomenological Langevin equation. This approach allows to properly take into account both the effects of the thermal bath on the system: a systematic dyssipative force, which tends to erase out any initial subcritical configuration, and a random stochastic force responsible for the fluctuations. We show that the contribution to the variance $\left\langle\left\langle\phi^{2}(t)\right\rangle\right\rangle_{V}$ in a given volume $V$ from any initial subcritical configuration is quickly damped away and that, in the limit of long times, $\left\langle\left\langle\phi^{2}(t)\right\rangle\right\rangle_{V}$ approaches its equilibrium value provided by the stochastic force and independent from the viscosity coefficient, as predicted by the fluctuation-dissipation theorem. In agreement with some recent claims, we conclude that thermal fluctuations do not affect the nucleation of critical bubbles at the onset of the electroweak phase transition making electroweak baryogenesis scenarios still a viable possibility to explain the primordial baryon asymmetry in the Universe.

\footnotetext{
${ }^{1}$ email: illuminati@mvxpd5.pd.infn.it

${ }^{2}$ email: riotto@tsmi19.sissa.it. Address after November 95: Theoretical Astrophysics Group, NASA/Fermilab, Batavia, IL60510, USA.
} 


\section{Introduction}

Nucleation of critical bubbles during a first order electroweak phase transition has received much attention since the discovery of the possibility for electroweak baryogenesis [1]. Indeed, one of the basic ingredients for the generation of the baryon asymmetry (apart from the requirement of baryon- and CP-violating interactions) is the presence of an out-of-equilibrium state [2] which, during the first order electroweak phase transition with supercooling, is attained by critical bubbles expanding in the thermal bath of the unbroken phase.

However, less attention has been paid to the environment where the critical bubble nucleation is supposed to occur. Since critical bubbles have a finite size, phase transitions are highly local phenomena. Fluctuations of the Higgs scalar field $\phi$ around the origin $\phi=0$ with spatial correlations comparable to the critical bubble size may be expected to be important for bubble nucleation. Also, if thermal fluctuations are too large, any perturbative scheme could break down. In such a situation, the prediction of a first order phase transition becomes suspect, with the possibility that the entire scenario of electroweak baryogenesis might be invalidated.

On the other hand, while the presence of thermal fluctuations in any thermodynamical system is undisputed, their role in the dynamics of weakly first order phase transitions is still controversial.

In ref. [3], it was first conjectured that statistical fluctuations around equilibrium may be described by spherically symmetric configurations roughly extending over a correlation volume, where the correlation length is given by the inverse temperature dependent mass of the Higgs field: $\xi(T)=m^{-1}(T)$.

These fluctuations are referred to as subcritical bubbles. Their amplitude 
was estimated in ref. [3], where it was concluded that they are dominant if the Higgs mass $M_{H}$ is larger than $\sim 80 \mathrm{GeV}$, whence the fraction which the asymmetric vacuum occupies in the neighborhood of the critical temperature becomes of the order of unity. Therefore it was concluded that critical bubbles cannot be generated due to the inhomogeneities of the background field.

In ref. [3], however, the continuous disappearance of the subcritical bubbles was not accounted for. This can happen in two ways: the subcritical bubbles, being unstable configurations, tend to shrink; they are also subject to constant thermal bombardment so that they may disappear simply because of thermal noise.

Following general principles and estimating the scalar field two-point function, i.e. the variance of a gaussian fluctuation distribution, computed in a correlation volume, Dine et al. [4] and Anderson [5] have later argued that subcritical bubbles do not affect the nucleation of critical bubbles in an appreciably way.

Subsequently, Gelmini and Gleiser [6] rekindled the issue adopting a different point of view based on modelling by a set of Boltzmann equations the evolution with time $t$ of the number density $n(R, t)$ of subcritical bubbles with a certain radius $R$.

Under a specific assumption about the form of the destruction rate due to thermal noise, they found that for Higgs masses below $\sim 55 \mathrm{GeV}$ the approach to equilibrium is dominated by shrinking. Unfortunately, for the interesting range of Higgs masses dictated by the experimental constraints coming from LEP, $M_{H}>60 \mathrm{GeV}[7]$, their analysis is inconclusive since the approximations adopted break down.

Recently, Enqvist et al. [8] re-estimated the amplitude, average size and formation rate of subcritical bubbles taking into account the crucial role played by thermalization. Their starting point was the the observation that at the microscopic level, the true origin of the dominant thermal fluctuation is the perpetual creation and annihilation of spherical subcritical bubbles. Thus one should identify the typical amplitude and size of these bubbles with the values estimated by a statistical ensemble averaging, instead of assuming, for instance, the size fixed and equal to the correlation length $\xi(T)$. 
They also observed that a large subcritical bubble should resemble the critical one around the critical temperature: when $R$ increases, the form of the subcritical bubble should deform smoothly to reproduce the critical configuration when $R=R_{c}, R_{c}$ being the critical radius. Thus, it was found that the average size $\langle R\rangle$ of the subcritical bubbles is much larger than the correlation length, and that the average amplitude $\left\langle\phi_{0}^{2}\right\rangle^{1 / 2}$ at the core is much smaller than any previous estimate.

These results led the authors of ref. [8] to conclude that thermal fluctuations do not hinder the electroweak first order phase transition. Of course, treating the size and the amplitude of subcritical bubbles as statistical degrees of freedom of an equilibrium ensamble at a fixed temperature $T$ does not allow to follow the time evolution of such degrees of freedom. Thus, since in the approach of ref. [8] there is no dynamics involved, $\langle R\rangle$ and $\left\langle\phi_{0}^{2}\right\rangle^{1 / 2}$ should be interpretated as the most probable initial conditions for any configuration describing a subcritical bubble generated at the time $t=0$.

Analogous conclusions to those of ref. [8] have been very recently obtained by Bettencourt [9], who, along the same line followed by Hindmarsh and Rivers [10] for the $\lambda \phi^{4}$ theory, computed the probability for fluctuations of the Standard Model Higgs field, averaged over a given spatial scale, to exceed a specified value. He found that the probability for the Higgs scalar field to fluctuate from the symmetric to the asymmetric minimum before the latter becomes stable is very small for Higgs masses of order of those of the $W^{ \pm}$ and $Z^{0}$ bosons, whereas the converse is more likely.

The aim of the present paper is to take a further step in the investigation of the role played by subcritical bubbles during the onset of the electroweak phase transition. In particular, we wish to present an analytic treatment of the dynamics of subcritical bubbles, described by a suitably coarse-grained configuration $\phi(\mathbf{x}, t)$, in a random environment.

Indeed, thermal fluctuations are the manifestation of the interaction between the system and the surrounding environment. The peculiarity of the thermal bath is that a virtually infinite number of degrees of freedom takes part in the exchanges of energy with the system. A familiar example [11] are the molecules of a gas or a fluid in which the system (the brownian particle) is embedded. It is well known that the impacts between the the system and 
the surrounding particles cause two effects: a systematic dissipative force (friction) and a random force, responsible for the fluctuations.

To describe the dynamics of subcritical fluctuations in a thermal bath around the homogeneous configuration $\phi=0$, we will then assume a classical Markovian Langevin equation

$$
\ddot{\phi}(\mathbf{x}, t)-\nabla^{2} \phi(\mathbf{x}, t)+\eta \dot{\phi}(\mathbf{x}, t)=-\frac{\partial V(\phi, T)}{\partial \phi}+\gamma(\mathbf{x}, t),
$$

where $V(\phi, T)$ is the potential associated to the the scalar field $\phi(\mathbf{x}, t)$ and $\eta$ is the viscosity coefficient which takes into account the dissipative effects.

The fluctuations are modelled by introducing a stochastic additive force (noise term) $\gamma(\mathbf{x}, t)$ characterized by a gaussian distribution with

$$
\begin{aligned}
\langle\gamma(\mathbf{x}, t)\rangle & =0 \\
\left\langle\gamma(\mathbf{x}, t) \gamma\left(\mathbf{x}^{\prime}, t^{\prime}\right)\right\rangle & =2 D \delta^{3}\left(\mathbf{x}-\mathbf{x}^{\prime}\right) \delta\left(t-t^{\prime}\right)
\end{aligned}
$$

where $D$ is the diffusion coefficient.

The Langevin equation (1) describing the dynamics of a subcritical bubble in contact with a thermal bath is similar to the Kardar-Parisi-Zhang equation [12] modelling the evolution of fluctuations around the average profile of a growing interface, which, in our case, is represented by the homogeneous configuration $\phi=0$.

Since the dynamics is expected to bring the system into equilibrium with the thermal bath at long times, the Langevin equation describes correctly the long-time dynamics and reproduce the correct equilibrium behaviour if the fluctuation-dissipation condition $D=\eta T$, expressing the common origin of dissipation and fluctuation, is satisfied [13].

Equation (1) naturally incorporates different crucial informations: i) subcritical bubbles are subject to constant thermal bombardment so that they can be rapidly thermalized and disappear with a relaxation rate $\eta$ (this was also accounted for in ref. [8] where, however, no dynamics was involved); ii) subcritical bubbles, being unstable configurations, tend to shrink; iii) the crucial role played by the noise term $\gamma(\mathbf{x}, t)$ responsible for fluctuations. It tends to contrast the damping term $\eta \dot{\phi}$ and determines the form of the fluctuation $\left\langle\phi^{2}(\mathbf{x}, t)\right\rangle$ at long times as well as its final equilibrium value.

A few comments are in order here about our choice of eq. (1). In writing it we have assumed that the system is Markovian: the correlation time scale 
for the noise is smaller than the typical relaxation time scale for the system. We have also assumed the noise to be additive.

Recent works indicates that one should expect departures from the Langevin equation written above [14], although the details are very sensitive to the model one starts with. For istance, in a $\lambda \phi^{4}$ theory, the Langevin equation describing the dynamics of the long wavelength modes evolving in the bath formed by the short wavelength modes is characterized by a colored and multiplicative noise and by a space-time dependent viscosity coefficient (see Gleiser and Ramos in [14]).

In this paper we will adopt eq. (1) as a first step. In fact, we do not expect that the nature of the thermal noise will change the final equilibrium properties of the system, even if the relaxation time-scales can be changed. Since the physical results will be related to the final equilibrium value of $\left\langle\phi^{2}(\mathbf{x}, t)\right\rangle$, we believe that they will be not affected by more complicated (even if more physical) representations of the coupling of the field $\phi(\mathbf{x}, t)$ to the thermal bath.

This point of view is also motivated by the independence of the statistical moments of the $\phi(\mathbf{x}, t)$ field at long times from the viscosity coefficient $\eta$, as implicit in the fluctuation-dissipation theorem.

For sake of clarity we have decided to address the issue of dynamics of subcritical bubbles in the electroweak theory after having described the same issue for simpler theories.

The paper is then organized as follows. In Subsection 2.1 we describe in details, for a free scalar field theory in the absence of thermal environments, the dynamics of a configuration representing an initial departure from the minimum energy equilibrium configuration. This allows us to evaluate the typical shrinking lifetime $\tau_{s h}$ of such a configuration.

In Subsection 2.2 we let the free scalar field be coupled to a thermal environment and follow the dynamics of the fluctuation at short and long times. We determine the asymptotic value of $\left\langle\phi^{2}(\mathbf{x}, t)\right\rangle$ in a given volume for $t \gg 1 / \eta$, showing explicitly that it is independent of time and equal to its equilibrium value.

In Section 3 we make use of the results obtained in Section 2 to describe, through a self-consistent Hartree approximation, the strength of the thermal 
fluctuations for the $\lambda \phi^{4}$ theory.

In Section 4 we present our results for the electroweak theory, from which one can conclude that subcritical bubbles do not affect nucleations of critical bubbles in a appreciably way.

We finally draw in Section 5 our conclusions and perspectives for future work.

\section{Free scalar field: linear dynamics}

\subsection{Free evolution at zero temperature}

In this Section we want to analyze the evolution of a spherically-symmetric configuration in the linear regime and absence of coupling to the thermal bath. We already know from Derrick's theorem [15], which forbids in four dimensions the existence of static, finite-energy configurations for models containing a simple real scalar field, that such configurations must be unstable and decay with a relaxation time $\tau_{s h}$, that we now want to estimate [16].

Given the quadratic mass term $\left(m^{2}>0\right)$

$$
V(\phi)=\frac{m^{2}}{2} \phi^{2}
$$

the associated Klein-Gordon equation is given by

$$
\ddot{\phi}(\mathbf{x}, t)-\nabla^{2} \phi(\mathbf{x}, t)+m^{2} \phi(\mathbf{x}, t)=0 .
$$

We model the initial deviation at $t=0$ from the homogeneous minimumenergy configuration $\phi=0$ by a gaussian shape of the type

$$
\begin{aligned}
& \phi(\mathbf{x}, 0)=\phi_{0} \mathrm{e}^{-|\mathbf{x}|^{2} / 2 R_{0}^{2}}, \\
& \dot{\phi}(\mathbf{x}, 0)=0,
\end{aligned}
$$

i.e. we imagine a deviation formed initially at rest with initial radius $R_{0}$ and amplitude $\phi_{0}$ at its core. Eq. (4) is most easily solved by examining the Fourier components

$$
\tilde{\phi}(\mathbf{q}, t)=\int d^{3} \mathbf{x} \mathrm{e}^{i \mathbf{q} \cdot \mathbf{x}} \phi(\mathbf{x}, t)
$$


which evolve according to

$$
\ddot{\tilde{\phi}}(\mathbf{q}, t)+\left(|\mathbf{q}|^{2}+m^{2}\right) \tilde{\phi}(\mathbf{q}, t)=0
$$

The solution of equation (7) is easily found to be

$$
\tilde{\phi}(\mathbf{q}, t)=A(\mathbf{q}) \cos \left(\sqrt{|\mathbf{q}|^{2}+m^{2}} t\right)+B(\mathbf{q}) \sin \left(\sqrt{|\mathbf{q}|^{2}+m^{2}} t\right) .
$$

The initial conditions described in eq. (5) fix $A(\mathbf{q})$ and $B(\mathbf{q})$

$$
\begin{aligned}
& A(\mathbf{q})=(2 \pi)^{3 / 2} \phi_{0} R_{0}^{3} \mathrm{e}^{-|\mathbf{q}|^{2} R_{0}^{2} / 2}, \\
& B(\mathbf{q})=0
\end{aligned}
$$

which, when plugged into eq. (8) allow us to investigate the behaviour of the bubble's core with time

$$
\phi(\mathbf{0}, t)=\sqrt{\frac{2}{\pi}} \phi_{0} R_{0}^{3} \int_{0}^{\infty} d|\mathbf{q}||\mathbf{q}|^{2} \mathrm{e}^{-|\mathbf{q}|^{2} R_{0}^{2} / 2} \cos \left(\sqrt{|\mathbf{q}|^{2}+m^{2}} t\right) .
$$

Since the integral is dominated by small values of $|\mathbf{q}|,|\mathbf{q}| \lesssim \sqrt{2} R_{0}^{-1}$, we can approximate the argument of $\cos \left(\sqrt{|\mathbf{q}|^{2}+m^{2}} t\right)$ for $R_{0} \gtrsim \sqrt{2} m^{-1}$ and write

$$
\begin{aligned}
\phi(\mathbf{0}, t) & \simeq \sqrt{\frac{2}{\pi}} \phi_{0} R_{0}^{3} \int_{0}^{\infty} d|\mathbf{q}||\mathbf{q}|^{2} \operatorname{Re}\left(\mathrm{e}^{i m t} \mathrm{e}^{-|\mathbf{q}|^{2} R_{0}^{2} / 2} \mathrm{e}^{i|\mathbf{q}|^{2} t / 2 m}\right) \\
& =\phi_{0} R_{0}^{3} \frac{\cos \left[m t+\frac{3}{2} \tan ^{-1}\left(t / m R_{0}^{2}\right)\right]}{\left(R_{0}^{4}+\frac{t^{2}}{m^{2}}\right)^{3 / 4}} .
\end{aligned}
$$

Thus, given an initial deviation from the stable configuration $\phi=0$ with sufficiently large initial size $R_{0}$ and in absence of coupling to a thermal bath, the amplitude at the core decays as $t^{-3 / 2}$ and the unstable configuration shrinks by radiating away its initial energy after a lifetime 16]

$$
\tau_{s h} \sim m R_{0}^{2} \gtrsim \frac{1}{m}
$$

\subsection{Coupling to the thermal bath}

The next step is to investigate how the dynamics of the sphericallysymmetric fluctuations around the homogeneous configuration $\phi=0$ changes when the system is coupled to a thermal bath. 
The Langevin equation (1) reads

$$
\ddot{\phi}(\mathbf{x}, t)-\nabla^{2} \phi(\mathbf{x}, t)+\eta \dot{\phi}(\mathbf{x}, t)+m^{2} \phi(\mathbf{x}, t)=\gamma(\mathbf{x}, t) .
$$

One can imagine the above equation to emerge from an effective theory where the fields (for instance, scalars different from $\phi(\mathbf{x}, t)$ or fermions) which $\phi(\mathbf{x}, t)$ is coupled to have been integrated out leaving an effective potential approximated at high temperature by eq. (3). In such a case $m^{2}$ has to be understood as a function of the temperature, $m^{2}(T) \sim g^{2} T^{2}, g$ being the generic coupling constant of $\phi(\mathbf{x}, t)$ to the other fields of the underlying original theory.

We wish to point out once more that the form of the Langevin equation arising from an effective theory is strongly model-dependent. Nevertheless, since we are interested in the equilibrium value of the fluctuation $\left\langle\phi^{2}(\mathbf{x}, t)\right\rangle$, we are confident that our result will not be affected by using eq. (13) to describe the dynamics of subcritical bubbles.

Again, eq. (13) is most easily solved by examining the Fourier components of the field $\phi(\mathbf{x}, t)$, see eq. (6). If we define

$$
\tilde{\gamma}(\mathbf{q}, t)=\int d^{3} \mathbf{x} \mathrm{e}^{i \mathbf{q} \cdot \mathbf{x}} \gamma(\mathbf{x}, t),
$$

the Fourier transformed noise, the equation to solve is

$$
\ddot{\tilde{\phi}}(\mathbf{q}, t)+\eta \dot{\tilde{\phi}}(\mathbf{q}, t)+\left(|\mathbf{q}|^{2}+m^{2}\right) \tilde{\phi}(\mathbf{q}, t)=\tilde{\gamma}(\mathbf{q}, t) .
$$

We define the function $\chi$ as

$$
\chi(\mathbf{q}) \equiv \eta^{2}-4\left(|\mathbf{q}|^{2}+m^{2}\right),
$$

and assume

$$
\eta<2 m
$$

The case $\eta>2 m$, though straightforward, is computationally more involved. On the other hand, it is physically the least interesting case, since any oscillatory behaviour is quickly dominated by the exponentially damped factors. Moreover, it obviously leads to the same results as far as the final equilibrium values are concerned.

The solution of eq. (15) can be cast into the form

$$
\tilde{\phi}(\mathbf{q}, t)=\tilde{\phi}_{h o}(\mathbf{q}, t)+\tilde{\phi}_{\gamma}(\mathbf{q}, t),
$$


where

$$
\begin{aligned}
\tilde{\phi}_{h o}(\mathbf{q}, t) & =A(\mathbf{q}) \mathrm{e}^{-\eta t / 2} \cos \frac{\sqrt{|\chi(\mathbf{q})|}}{2} t+B(\mathbf{q}) \mathrm{e}^{-\eta t / 2} \sin \frac{\sqrt{|\chi(\mathbf{q})|}}{2} t \\
\tilde{\phi}_{\gamma}(\mathbf{q}, t) & =-2 \mathrm{e}^{-\eta t / 2} \cos \frac{\sqrt{|\chi(\mathbf{q})|}}{2} t \int_{0}^{t} d \tau \frac{\mathrm{e}^{\eta \tau / 2}}{\sqrt{|\chi(\mathbf{q})|}} \sin \frac{\sqrt{|\chi(\mathbf{q})|}}{2} \tau \gamma(\mathbf{q}, \tau) \\
& +2 \mathrm{e}^{-\eta t / 2} \sin \frac{\sqrt{|\chi(\mathbf{q})|}}{2} t \int_{0}^{t} d \tau \frac{\mathrm{e}^{\eta \tau / 2}}{\sqrt{|\chi(\mathbf{q})|}} \cos \frac{\sqrt{|\chi(\mathbf{q})|}}{2} \tau \gamma(\mathbf{q}, \tau) .
\end{aligned}
$$

Note that $\tilde{\phi}_{\gamma}(\mathbf{q}, t)$ does not depend upon the initial conditions described by eq. (5), while they are embodied in $\tilde{\phi}_{h o}(\mathbf{q}, t)$ through the amplitudes $A(\mathbf{q})$ (given by eq. (9)) and

$$
B(\mathbf{q})=\frac{\eta}{\sqrt{|\chi(\mathbf{q})|}} A(\mathbf{q})
$$

When computing the mean values $\langle\cdot \cdot\rangle$ with respect to the gaussian measure of the noise $\gamma(\mathbf{x}, t)$, some care is needed. Indeed, as noted in the Introduction, one has to assume that the correlation time scale for the noise $\tau_{\gamma}$ is smaller than the typical relaxation time of the free system $\tau_{s h} \simeq R_{0}^{2} m$ as determined in Subsect. 2.1.

In the case under examination, $\eta<2 m$, this is true only for configurations with very large initial radius $R_{0} \gg m^{-1}$; then, from now on we will assume this to be the case $\}^{\text {j }}$, i.e. $\tau_{\gamma} \ll \tau_{s h}$.

From eq. (19) we see that fluctuations in each mode decay with a relaxation time given by $\tau_{\gamma} \simeq 1 / \eta$. This implies for the following decay law for the average values:

$$
\langle\tilde{\phi}(\mathbf{q}, t)\rangle \sim \exp (-\eta t / 2)
$$

as expected from the presence of the damping term in the Langevin equation. This is similar to what happens for a particle thrown into a thermal bath with an initial velocity $\vec{v}_{0}$ : after a sufficiently long time, and independently from the values of $\vec{v}_{0}$, the particle behaves like a brownian system

\footnotetext{
${ }^{3}$ Of course, in the opposite limit $\eta>2 m$, the correlation time scale for the noise is much smaller than any other time scales and no additional condition on $R_{0}$ is required.
} 
with a configurational average $\langle\vec{X}\rangle=0$ and a nonvanishing fluctuation $\left\langle\vec{X}^{2}\right\rangle$ determined by the surrounding noise.

The two-point correlation function at equal time is given by

$$
\begin{aligned}
\left\langle\phi(\mathbf{x}, t) \phi\left(\mathbf{x}^{\prime}, t\right)\right\rangle & =\int \frac{d^{3} \mathbf{q}}{(2 \pi)^{3}} \mathrm{e}^{-i \mathbf{q} \cdot \mathbf{x}} \int \frac{d^{3} \mathbf{q}^{\prime}}{(2 \pi)^{3}} \mathrm{e}^{-i \mathbf{q}^{\prime} \cdot \mathbf{x}^{\prime}}\left\langle\tilde{\phi}(\mathbf{q}, t) \tilde{\phi}\left(\mathbf{q}^{\prime}, t\right)\right\rangle \\
& =\phi_{h o}(\mathbf{x}, t) \phi_{h o}\left(\mathbf{x}^{\prime}, t\right)+\left\langle\phi(\mathbf{x}, t) \phi\left(\mathbf{x}^{\prime}, t\right)\right\rangle_{\gamma}
\end{aligned}
$$

where we have defined

$$
\left\langle\phi(\mathbf{x}, t) \phi\left(\mathbf{x}^{\prime}, t\right)\right\rangle_{\gamma}=\int \frac{d^{3} \mathbf{q}}{(2 \pi)^{3}} \mathrm{e}^{-i \mathbf{q} \cdot \mathbf{x}} \int \frac{d^{3} \mathbf{q}^{\prime}}{(2 \pi)^{3}} \mathrm{e}^{-i \mathbf{q}^{\prime} \cdot \mathbf{x}^{\prime}}\left\langle\tilde{\phi}_{\gamma}(\mathbf{q}, t) \tilde{\phi}_{\gamma}\left(\mathbf{q}^{\prime}, t\right)\right\rangle .
$$

Making use of the two-point correlation function for the Fourier transformed noise

$$
\left\langle\gamma(\mathbf{q}, t) \gamma\left(\mathbf{q}^{\prime}, t^{\prime}\right)\right\rangle=2(2 \pi)^{3} \eta T \delta^{3}\left(\mathbf{q}+\mathbf{q}^{\prime}\right) \delta\left(t-t^{\prime}\right),
$$

eq. (23) becomes

$$
\begin{aligned}
\left\langle\phi(\mathbf{x}, t) \phi\left(\mathbf{x}^{\prime}, t\right)\right\rangle_{\gamma} & =4 T \int \frac{d^{3} \mathbf{q}}{(2 \pi)^{3}} \mathrm{e}^{-i \mathbf{q} \cdot\left(\mathbf{x}-\mathbf{x}^{\prime}\right)} \frac{1}{|\chi(\mathbf{q})|}\left[\frac{|\chi(\mathbf{q})|}{\eta^{2}+|\chi(\mathbf{q})|}-\mathrm{e}^{-\eta t}\right. \\
& \left.+\mathrm{e}^{-\eta t} \frac{\eta^{2} \cos \sqrt{|\chi(\mathbf{q})|} t-\eta \sqrt{|\chi(\mathbf{q})|} \sin \sqrt{|\chi(\mathbf{q})|} t}{\eta^{2}+|\chi(\mathbf{q})|}\right] .
\end{aligned}
$$

We are now in the position to study the evolution with time of the magnitude of the fluctuation around the $\phi=0$ state. A natural measure of it is given by the two-point connected Green's function, coarse-grained on a volume $V$, defined as

$$
\begin{aligned}
\left\langle\left\langle\phi^{2}(t)\right\rangle\right\rangle_{V} & =\frac{1}{V^{2}} \int d^{3} \mathbf{x} \int d^{3} \mathbf{x}^{\prime}\left\langle\phi(\mathbf{x}, t) \phi\left(\mathbf{x}^{\prime}, t\right)\right\rangle I(\mathbf{x}) I\left(\mathbf{x}^{\prime}\right) \\
& =\left\langle\left\langle\phi^{2}(t)\right\rangle\right\rangle_{V}^{h o}+\left\langle\left\langle\phi^{2}(t)\right\rangle\right\rangle_{V}^{\gamma},
\end{aligned}
$$

where the definition of $\left\langle\left\langle\phi^{2}(t)\right\rangle\right\rangle_{V}^{h o}$ and $\left\langle\left\langle\phi^{2}(t)\right\rangle\right\rangle_{V}^{\gamma}$ can be easily read from eq. (22) and

$$
I(\mathbf{x})=\sqrt{\frac{2}{9 \pi}} \mathrm{e}^{-|\mathbf{x}|^{2} / 2 R^{2}}
$$


is a window function modelling the volume $V=(4 \pi / 3) R^{3}$ over which we test the magnitude of the fluctuationst

The system as a whole is taken to have a volume much larger than $V$. If we denote by $\xi \simeq 1 / m$ the correlation length, we can parametrize the number of correlation volumes by writing $R=\beta \xi$, where $\beta$ is a positive number. Since, at any time, the field $\phi$ is correlated over some correlation length, it will be sufficient to take $\beta>1$.

From eq. (26) we see that the variance $\left\langle\left\langle\phi^{2}(t)\right\rangle\right\rangle_{V}$ receives two contributions: $\left\langle\left\langle\phi^{2}(t)\right\rangle\right\rangle_{V}^{h o}$ describing the contribution coming from the gaussian configuration formed at $t=0$ and $\left\langle\left\langle\phi^{2}(t)\right\rangle\right\rangle_{V}^{\gamma}$ expressing the contribution due to the noise force. As a consequence, the latter does not depend on the initial conditions.

Let us now imagine that the gaussian configuration (5) represents a deviation from the equilibrium value formed at $t=0$ due to thermal fluctuations. To follow the dynamics of $\left\langle\left\langle\phi^{2}(t)\right\rangle\right\rangle_{V}$ we can then identify two different time regimes:

\section{i) Short times:}

For $t \ll 1 / \eta$, none of the modes of $\left\langle\left\langle\phi^{2}(t)\right\rangle\right\rangle_{V}^{h o}$ has yet relaxed, but $\left\langle\left\langle\phi^{2}(t)\right\rangle\right\rangle_{V}^{h o}$ starts to decrease as $t^{2}$, that is

$$
\begin{gathered}
\left\langle\left\langle\phi^{2}(t)\right\rangle\right\rangle_{V}^{h o}-\left\langle\left\langle\phi^{2}(0)\right\rangle\right\rangle_{V}^{h o} \simeq \\
-t^{2} \int \frac{d^{3} \mathbf{q}}{(2 \pi)^{3}} \mathrm{e}^{-|\mathbf{q}|^{2} R^{2} / 2} A(\mathbf{q})\left(|\mathbf{q}|^{2}+m^{2}\right) \int \frac{d^{3} \mathbf{q}^{\prime}}{(2 \pi)^{3}} \mathrm{e}^{-\left|\mathbf{q}^{\prime}\right|^{2} R^{2} / 2} A\left(\mathbf{q}^{\prime}\right)= \\
-t^{2} \frac{\phi_{0}^{2} R_{0}^{6}}{\left(R_{0}^{2}+R^{2}\right)^{3}}\left(m^{2}+\frac{3}{R_{0}^{2}+R^{2}}\right) .
\end{gathered}
$$

Taking $R_{0} \simeq R=\beta \xi$ and $\beta \gg 1$, we see that the contribution to $\left\langle\left\langle\phi^{2}(t)\right\rangle\right\rangle_{V}$ coming from the gaussian configuration formed at $t=0,\left\langle\left\langle\phi^{2}(t)\right\rangle\right\rangle_{V}^{h_{o}}$, decreases as $\sim t^{2} \phi_{0}^{2} m^{2}$.

\footnotetext{
${ }^{4}$ The coefficient $\sqrt{2 / 9 \pi}$ in eq. $(27)$ is chosen to make easier the comparison between our results in Section 4 and those in ref. [9] in the case of the fluctuations at the onset of the electroweak phase transition.
} 
In the meanwhile, the contribution to $\left\langle\left\langle\phi^{2}(t)\right\rangle\right\rangle_{V}$ from the noise force, $\left\langle\left\langle\phi^{2}(t)\right\rangle\right\rangle_{V}^{\gamma}$, starts to increase as $t^{3}$ :

$$
\begin{aligned}
\left\langle\left\langle\phi^{2}(t)\right\rangle\right\rangle_{V}^{\gamma} & \simeq \frac{2 \eta T}{3} t^{3} \int \frac{d^{3} \mathbf{q}}{(2 \pi)^{3}} \mathrm{e}^{-|\mathbf{q}|^{2} R^{2}} \\
& =\frac{1}{12 \pi^{3 / 2}} \frac{\eta T}{R^{3}} t^{3} .
\end{aligned}
$$

ii) Long times:

For $t \gg 1 / \eta$, all the modes have relaxed to their equilibrium values. The initial gaussian configuration (5) has been rapidly thermalized with a relaxation time $\tau_{\gamma} \simeq 1 / \eta$ so that $\left\langle\left\langle\phi^{2}(t)\right\rangle\right\rangle_{V}^{h o}$ has quickly vanished.

The situation is again similar to that for a brownian particle immersed in a thermal environment at $t=0$ with initial data $\vec{X}_{0}, \vec{v}_{0}$ : the contribution to $\left\langle\vec{X}^{2}(t)\right\rangle$ due to the initial conditions is very rapidly dissipated away and taken over by the contribution from the noise.

As a consequence, at long times $\left\langle\left\langle\phi^{2}(t)\right\rangle\right\rangle_{V}^{\gamma}$ provides the only contribution to $\left\langle\left\langle\phi^{2}(t)\right\rangle\right\rangle_{V}$ which tends asymptotically towards the time-independent dynamical value

$$
\left\langle\left\langle\phi^{2}\right\rangle\right\rangle_{V}^{\text {dyn }}=\int \frac{d^{3} \mathbf{q}}{(2 \pi)^{3}} \mathrm{e}^{-|\mathbf{q}|^{2} R^{2}} \frac{T}{|\mathbf{q}|^{2}+m^{2}} .
$$

Note that the dependence from the the viscosity coefficient $\eta$ in the above expression is absent as implicit in the fluctuation-dissipation theorem: the system after a sufficiently long time relaxes towards its equilibrium state which is completely independent from $\eta$.

It is readily verified that the above expression coincides with the equilibrium value of the two-point connected Green's function, coarse-grained on a volume $V$, calculated in ref. [10]. This is not surprising. We already know that dynamics is expected to bring the thermal fluctuations represented by the subcritical bubbles into equilibrium with the surrounding thermal bath at long times.

The result expressed by eq. (30) shows explicitly that the Langevin equation correctly describes the long-time behaviour of $\left\langle\left\langle\phi^{2}(t)\right\rangle\right\rangle_{V}$ reproducing its equilibrium value if the fluctuation-dissipation condition is satisfied, i.e

$$
\lim _{t \rightarrow \infty}\left\langle\left\langle\phi^{2}(t)\right\rangle\right\rangle_{V} \equiv\left\langle\left\langle\phi^{2}\right\rangle\right\rangle_{V}^{\mathrm{dyn}}=\left\langle\left\langle\phi^{2}\right\rangle\right\rangle_{V}^{\mathrm{EQ}}
$$


Note that eq. (30) still holds also in the case $\eta>2 m$. Namely, in the overdamped limit (large times or strong coupling to the environment), the description simplifies considerably, as the canonical momentum associated to $\phi(\mathbf{x}, t)$ becomes a variable much faster than the field itself and can be considered fully thermalized in the adiabatic approximation.

The above statement amounts to drop the inertia term $\ddot{\phi}(\mathbf{x}, t)$ in eq. (15), leading to the equation

$$
\eta \dot{\tilde{\phi}}(\mathbf{q}, t)+\left(|\mathbf{q}|^{2}+m^{2}\right) \tilde{\phi}(\mathbf{q}, t)=\tilde{\gamma}(\mathbf{q}, t)
$$

which reproduces the large time behaviour expressed by eq. (30).

Assuming $R=\beta / m$ and noting that the gaussian function in eq. (30) is substantially different from zero only for $|\mathbf{q}|^{2} \lesssim m^{2} / \beta^{2}$, we get

$$
\left\langle\left\langle\phi^{2}\right\rangle\right\rangle_{V}^{\mathrm{dyn}} \simeq \frac{m T}{2 \pi^{2}}\left[\frac{1}{\beta}-\tan ^{-1}\left(\frac{1}{\beta}\right)\right] .
$$

The spreading of the field over a correlation volume $V_{\xi}=(4 \pi / 3) \xi^{3}$ is then

$$
\left\langle\left\langle\phi^{2}\right\rangle\right\rangle_{V_{\xi}}^{\mathrm{dyn}} \simeq 10^{-2} m T
$$

For volumes $V$ substantially larger than the correlation volume, $\left\langle\left\langle\phi^{2}\right\rangle\right\rangle_{V}^{\text {dyn }} \simeq$ $\left(m T / 3 \pi^{2} \beta^{3}\right) \ll m T$. The coefficient $1 / 2 \pi^{2}$ plays a crucial role in reducing the field spreading, as already pointed out in refs. [4, 9, 10].

One could expect from these results that subcritical fluctuations will not play a significant role in the electroweak phase transition. Nevertheless, all the considerations made in this Section are limited to a linear system. In the next Section we wish to extend them, through a self-consistent Hartree approximation, to a nonlinear system described by a $\lambda \phi^{4}$ potential as a further step torwards the study of thermal fluctuations at the onset of electroweak phase transition.

\section{Self-interacting field: Hartree approximation}

The above description of the evolution of spherically-symmetric initial configurations in the presence of a thermal bath is based on the linearity of the Langevin equation. 
Let us now consider the issue of thermal fluctuations around the homogeneous configuration $\phi=0$ for a nonlinear system with a potential

$$
V(\phi)=\frac{m^{2}}{2} \phi^{2}+\frac{\lambda}{4} \phi^{4}
$$

where $m^{2}$ is assumed to be positive so that $V(\phi)$ posseses a unique minimum at $\phi=0$.

In order to put the theory in a form suitable for the application of the formalism developed in Section 2, we first resort to the Hartree approximation. Taking the expectations over the free Gaussian measure with their proper haffnian combinatoric factor, the Hartree prescription yields the following substitution:

$$
\phi^{4}(\mathbf{x}, t) \rightarrow 6\left\langle\left\langle\phi^{2}\right\rangle\right\rangle_{V}^{\text {dyn }} \phi^{2}(\mathbf{x}, t)
$$

where $\left\langle\left\langle\phi^{2}\right\rangle\right\rangle_{V}^{\text {dyn }}$ is the asymptotic, time-independent value of the two-point connected Green's function, to be determined self-consistently by equating its expression to the equilibrium value $\left\langle\left\langle\phi^{2}\right\rangle\right\rangle_{V}^{\mathrm{EQ}}$ derived from the dynamics.

In the Hartree theory the potential $V(\phi)$ is then substituted by an effective linear potential

$$
V_{\mathrm{eff}}=\frac{m_{H}^{2}}{2} \phi^{2}
$$

where the Hartree effective mass is now given by

$$
m_{H}^{2}=m^{2}+3 \lambda\left\langle\left\langle\phi^{2}\right\rangle\right\rangle_{V}^{\text {dyn }}
$$

The fact that $m_{H}^{2}$ is time-independent allows us to solve the eq. (13) with the substitution $m^{2} \rightarrow m_{H}^{2}$ and to make use of the results given in Subsection 2.2. The contribution to $\left\langle\left\langle\phi^{2}(t)\right\rangle\right\rangle_{V}$ from the initial configuration is expected to be dissipated away within a typical relaxation time $\tau_{\gamma}$, whereas the contribution from the stochastic force increases with time reaching for $t \gg 1 / \eta$ the asymptotic value $\left\langle\left\langle\phi^{2}\right\rangle\right\rangle_{V}^{\text {dyn }}$.

From eq. (30) one can easily read off the self-consistency condition as an intersection equation:

$$
\begin{aligned}
\left\langle\left\langle\phi^{2}\right\rangle\right\rangle_{V}^{\text {dyn }} & =f\left(\lambda,\left\langle\left\langle\phi^{2}\right\rangle\right\rangle_{V}^{\text {dyn }}\right) \equiv \int \frac{d^{3} \mathbf{q}}{(2 \pi)^{3}} \mathrm{e}^{-|\mathbf{q}|^{2} R^{2}} \frac{T}{|\mathbf{q}|^{2}+m_{H}^{2}} \\
& =\int \frac{d^{3} \mathbf{q}}{(2 \pi)^{3}} \mathrm{e}^{-|\mathbf{q}|^{2} R^{2}} \frac{T}{|\mathbf{q}|^{2}+m^{2}+3 \lambda\left\langle\left\langle\phi^{2}\right\rangle\right\rangle_{V}^{\text {dyn }}} .
\end{aligned}
$$


An upper bound for $\left\langle\left\langle\phi^{2}\right\rangle\right\rangle_{V}^{\text {dyn }}$ then is trivially found observing that

$$
f\left(\lambda,\left\langle\left\langle\phi^{2}\right\rangle\right\rangle_{V}^{\mathrm{dyn}}\right) \leq f\left(0,\left\langle\left\langle\phi^{2}\right\rangle\right\rangle_{V}^{\mathrm{dyn}}\right)
$$

which, taking into account the results of the previous Section, assures that

$$
\left\langle\left\langle\phi^{2}\right\rangle\right\rangle_{V}^{\mathrm{dyn}} \lesssim 10^{-2} m T \text {. }
$$

This inequality is not surprising: being $m^{2}>0$, a positive value of $\lambda$ has the effect to increase the effective mass around $\phi=0$ making fluctuations more difficult than in the case $\lambda=0$.

Some comments are in order here. First of all, one can expect the Hartree approximation to be reliable in the limit of small coupling $\lambda$, i.e. when nonlinear effects can be neglected in first approximation. In this respect, the Hartree approximation amounts to consider the scalar field $\phi$ to be the modulus of a vector field $\vec{\varphi}$ with a large number $N$ of components and selfcoupling $(\lambda / N)|\vec{\varphi}|^{4}$ [17.

We have considered a nonlinear potential (35), that does not depend upon the volume $V$ of the region where one takes the space average of the variance at long times.

This is strictly correct [10] only for regions with typical size $R \gtrsim \xi$ which are the ones we are interested in. For $R \lesssim \xi$, one should modify expression (35) to properly take into account the finite size effects.

In the next Section we finally address the role of thermal fluctuations in the physically interesting case of the electroweak phase transition and investigate whether the latter is hindered by the presence and the equilibrium dynamics of the subcritical bubbles.

\section{Fluctuations at the electroweak phase transition}

\subsection{Small supercooling limit and thin wall approximation}

First order phase transition and critical bubble dynamics in the Standard Model have lately been studied in much detail, and it has become increasingly clear 18 that for Higgs masses considerably heavier than $60 \mathrm{GeV}$, the electroweak phase transition is only of weakly first order. 
For a Higgs mass $M_{H}>100 \mathrm{GeV}$, both perturbative and lattice calculations confront technical problems. However, it is conceivable that for such large Higgs masses the electroweak phase transition is close to a second order and does not proceed by critical bubble formation.

In this Section we consider a phenomenological Higgs potential for the order parameter $\phi$ suitable for a simple description of a first order phase transition:

$$
V(\phi)=\frac{1}{2} m^{2}(T) \phi^{2}-\frac{1}{3} \alpha T \phi^{3}+\frac{1}{4} \lambda \phi^{4}
$$

where we have not determined the parameters perturbatively but fit them, when needed, according to a recent two-loop determination of the gaugeinvariant effective potential [19].

Most of the dynamical properties of the electroweak phase transition associated with the potential Eq. (42), such as the smallness of the latent heat, the bubble nucleation rate and the size of critical bubbles, have been discussed in [20]. For the purposes of the present paper it suffices to recall only some of the results.

Assuming that there is only little supercooling, as seems to be the case for the electroweak phase transition, the bounce action can be written as

$$
S / T=\frac{\alpha}{\lambda^{3 / 2}} \frac{2^{9 / 2} \pi}{3^{5}} \frac{\bar{\lambda}^{3 / 2}}{(\bar{\lambda}-1)^{2}},
$$

where $\bar{\lambda}(T)=9 \lambda m^{2}(T) /\left(2 \alpha^{2} T^{2}\right)$. The cosmological transition temperature is determined from the relation that the Hubble rate equals the transition rate $\propto e^{-S / T}$, yielding $S / T_{f} \simeq \ln \left(M_{P l}^{4} / T_{f}^{4}\right) \simeq 150$, where $T_{f}$ is the transition temperature. Thus we obtain from Eq. (43)

$$
\bar{\lambda}\left(T_{f}\right) \simeq 1-0.0442 \frac{\alpha^{1 / 2}}{\lambda^{3 / 4}} \equiv 1-\delta .
$$

On the other hand, small supercooling implies that $1-\bar{\lambda}=\delta \ll 1$, i.e. $\alpha \ll 500 \lambda^{3 / 2}$. Solving for $\bar{\lambda}$ in Eq. (43) yields the transition temperature $T_{f}$. One finds

$$
m^{2}\left(T_{f}\right)=\frac{2 \alpha^{2}}{9 \lambda} \bar{\lambda}\left(T_{f}\right) T_{f}^{2} .
$$

The extrema of the potential are given by

$$
\phi_{ \pm}(T)=\frac{\alpha T}{2 \lambda}(1 \pm \sqrt{1-8 \bar{\lambda} / 9})
$$


Expanding the potential at the broken minimum $\phi_{+}(T)$ we find

$$
-\epsilon \equiv V\left(\phi_{+}, T_{f}\right)=\frac{1}{6} m^{2}\left(T_{f}\right) \phi_{+}^{2}-\frac{1}{12} \lambda \phi_{+}^{4}=-0.00218 \frac{\alpha^{9 / 2}}{\lambda^{15 / 4}} T_{f}^{4}+\mathcal{O}\left(\delta^{2}\right) \text {. }
$$

The height of the barrier is situated at $\phi_{-} \simeq \phi_{+} / 2$ with $V\left(\phi_{-}, T_{c}\right) \equiv V_{\max }=$ $\alpha^{4} T_{c}^{4} /\left(144 \lambda^{3}\right)$, where $T_{c}$ is the temperature at which $V(0)=V\left(\phi_{+}\right)$, given by the condition $m\left(T_{c}\right)^{2}=\left(2 \alpha^{2} T_{c}^{2} / 9 \lambda\right)$. As $T_{c} \simeq T_{f}$ we may conclude that the thin wall approximation is valid if $-\epsilon / V_{\max }=0.314 \alpha^{1 / 2} / \lambda^{3 / 4} \ll 1$, or $\alpha \ll 10 \lambda^{3 / 2}$. Thus the small supercooling limit is clearly satisfied if the thin wall approximation is valid.

At $T=T_{f}$ the system can tunnel from the unbroken phase $\phi=0$ to the broken phase $\phi=\phi_{+}$throught the formation of energetically favoured critical bubbles with critical radius $R_{c}$.

To get the size of the critical bubble we still need the surface tension. One easily finds

$$
\sigma=\int_{0}^{\infty} d \phi \sqrt{2 V\left(T_{c}\right)}=\frac{2 \sqrt{2} \alpha^{3}}{91 \lambda^{5 / 2}} T_{c}^{3}
$$

We define the critical bubble radius by extremizing the bounce action. In the thin wall approximation the result is

$$
R_{c}=13.4 \frac{\lambda^{3 / 4}}{\alpha^{1 / 2} m\left(T_{f}\right)} .
$$

Therefore $R_{c}$ is much larger than the correlation length $\xi\left(T_{f}\right)=1 / m\left(T_{f}\right)$ at the transition temperature, as it should. It is clear that fluctuations of the Higgs scalar field $\phi$ with spatial correlations comparable to the critical bubble size may be expected to be important for bubble nucleation.

\subsection{Subcritical bubbles}

Let us first make the general observation that it is the actual transition temperature $T_{f}$ rather than the critical temperature $T_{c}$ which is relevant in the study of subcritical bubbles. This is true in the sense that if subcritical bubbles are not important at $T_{f}$, they will not be at $T_{c}$ either.

As we shall show, it actually turns out that subcritical bubbles are not important even at $T_{f}$. This justifies, in retrospect, our choice $T=T_{f}$ for performing the calculations. 
In the case of a weak first order phase transition the critical bubble is typically well described by a thin wall approximation, where the configuration is by no means gaussian, but has a flat 'highland' (with $\phi$ determined by the non-zero minimum of the potential) and a steep slope down to $\phi=0$.

Therefore it seems natural that also a large subcritical bubble should resemble the critical one: when its radius $R$ increases, the form of the subcritical bubble should deform smoothly so that, when $R=R_{c}$, the bubble is a critical one.

The authors of ref. [8] took into account this observation in their choice of the ansatz $\phi=\phi\left(\phi_{0}, R\right)$ for the configuration describing the thermal fluctuations around equilibrium.

Treating the amplitude $\phi_{0}$ (at the core) and the radius $R$ of such fluctuation as statistical degrees of freedom, they found that the average size $\langle R\rangle$ is much larger than the correlation length $\xi\left(T_{f}\right)$ and that the most probable amplitude at the core $\left\langle\phi_{0}^{2}\right\rangle^{1 / 2}$ is much smaller than $\phi_{-}\left(T_{f}\right)$, the value of the field $\phi$ where the potential acquires its maximum.

It was then concluded [8] that subcritical bubbles, at least for Higgs masses less than about $100 \mathrm{GeV}$, are not important during the onset of the electroweak phase transition.

This conclusion was drawn also by taking into account the crucial role played by thermalization: since the thermalization rate $\eta$ for small amplitude scalar fluctuations and large spatial size was estimated in the Standard Model [21] to be of order of $10^{-2} T$ near the critical temperature, i.e. much larger than the typical first order transition time, small amplitude fluctuations with size larger than about $1 / \eta$ were considered to be absent from the mixture of subcritical bubbles and not counted in the thermal averages.

However, we know that the fluctuation-dissipation theorem describes the common origin of dissipation and fluctuation: the impact between the system and the surrounding particles of the thermal bath is not limited to a systematic friction force responsible for the dissipation of fluctuations, but is also characterized by a random force responsible for the fluctuations themselves. This stochastic force is fundamental, as we have seen in Section 2, in determining the dynamics of fluctuations with time and expecially in the asymptotic regime. 
From the considerations above, it should be clear that the thermal averages $\langle R\rangle$ and $\left\langle\phi_{0}^{2}\right\rangle^{1 / 2}$ found in ref. [8 must be respectively regarded as the most probable size and amplitude at the core of a subcritical bubble formed at $t=0$. Let us then imagine that this "most probable" configuration appears at $t=0$.

To follow its dynamics, again we linearize the potential applying the Hartree approximation:

$$
\phi^{3}(\mathbf{x}, t) \rightarrow 3\left\langle\left\langle\phi^{2}\right\rangle\right\rangle_{V}^{\text {dyn }} \phi(\mathbf{x}, t), \quad \phi^{4}(\mathbf{x}, t) \rightarrow 6\left\langle\left\langle\phi^{2}\right\rangle\right\rangle_{V}^{\text {dyn }} \phi^{2}(\mathbf{x}, t) .
$$

The linearized Langevin equation now reads

$$
\ddot{\phi}(\mathbf{x}, t)-\nabla^{2} \phi(\mathbf{x}, t)+\eta \dot{\phi}(\mathbf{x}, t)+m_{H}^{2} \phi(\mathbf{x}, t)-\alpha T\left\langle\left\langle\phi^{2}\right\rangle\right\rangle_{V}^{\text {dyn }}=\gamma(\mathbf{x}, t),
$$

where $m_{H}^{2}$ is the same in equation (38).

Since we are assuming the electroweak phase transition to be first order, which is expected to occur for for light Higgs masses or equivalently $\lambda \ll 1$, and since thermal fluctuations are expected to be important for a very weak first order phase transition, i.e. for $\alpha \ll 1$, we expect the Hartree approximation for the quartic potential (42) to be reliable since the coefficients of the nonlinear terms in the potential are very small.

The Hartree approximation does not allow to describe the nonlinear effects present in the theory. For instance, Copeland et al. [16 have recently pointed out that nonlinear scalar field theories can incorporate well localized, time-dependent, configurations, called oscillons, which, although unstable, can be extremely long-lived, $\tau_{s h} \simeq\left(10^{3}-10^{4}\right) m^{-1}$. However, such configurations can develop resonances only if they form with an initial amplitude $\phi_{0}$ at the core above the inflection point $\phi_{-}$, which seems to be not very probable [8]. Moreover, oscillons were studied in absence of any coupling to a thermal bath whose presence is expected to reduce considerably their lifetime.

Equation (51) is far from being the true Langevin equation describing a thermal fluctuation in the hot electroweak theory. Indeed, even in the simpler $\lambda \phi^{4}$ theory, the noise is colored and the coupling to the thermal bath is multiplicative.

Also, the viscosity coefficient $\eta$ is not expected to be a constant, but to depend upon the typical momentum scale of the thermal fluctuation, i.e. the 
inverse of its radius, and its amplitude. Up to now the only estimate of $\eta$ for the Standard Model is that given in ref. [21] and is valid only for small amplitude $\phi_{0}$ and large size $R$ fluctuations, $R \gtrsim 1 / \eta \simeq 10^{2} / T$.

Fortunately enough, this is just the range of $\phi_{0}$ and $R$ provided in ref. [8] for the initial conditions that we have to specify for solving the linearized Langevin equation; we therefore expect that assuming a constant $\eta$ in momentum space provides a reliable approximation.

Again, physical results at long times, or at equilibrium, are obviously expected to be independent from the viscosity coefficient $\eta$ and from the nature of the thermal noise, even if relaxation times can change significantly.

Equation (51) is easily reduced in a form equivalent to the one holding in the free field case by absorbing the constant non-homogenous term through the following linear redefinition of the field variable:

$$
\begin{aligned}
\phi(\mathbf{x}, t) & =\varphi(\mathbf{x}, t)+\kappa, \\
\kappa & =\frac{\alpha T}{3 \lambda+\left(m_{H}^{2} /\left\langle\left\langle\phi^{2}\right\rangle\right\rangle_{V}^{\text {dyn }}\right)},
\end{aligned}
$$

where $\varphi(\mathbf{x}, t)$ solves the Langevin equation (13) with $m^{2} \rightarrow m_{H}^{2}$. Note that $\kappa$ in the limit of very large $\left\langle\left\langle\phi^{2}\right\rangle\right\rangle_{V}^{\text {dyn }}$ approaches $\phi_{-}^{2}$.

For definiteness, we fitted our phenomenological potential Eq. (42) to the two loop result for the effective potential calculated in [19] for the Higgs mass $M_{H}=70 \mathrm{GeV}$. This yields $\alpha \simeq 0.048$ and $\lambda \simeq 0.061$. One readily verifies that we are indeed safely in the thin wall limit. With these parameter values (in units of $R_{c}$ )

$$
\begin{aligned}
T_{f} & =85.70, \\
m^{2}\left(T_{f}\right) & =56.78 \\
\eta & \simeq 10^{-2} T_{f} \simeq 0.875 \\
\phi_{-}\left(T_{f}\right) & \simeq 24 .
\end{aligned}
$$

Assuming to be again in the situation $\eta<2 m_{H}$ (we shall verify a posteriori that this is indeed the case), we easily read off the solution of the linearized Langevin eq. (51):

$$
\phi(\mathbf{x}, t)=\varphi(\mathbf{x}, t)+\kappa\left(1-\mathrm{e}^{-\eta t / 2} \cos \frac{\sqrt{\eta^{2}-4 m_{H}^{2}}}{2} t\right.
$$




$$
\left.-\mathrm{e}^{-\eta t / 2} \frac{\eta}{\sqrt{\eta^{2}-4 m_{H}^{2}}} \sin \frac{\sqrt{\eta^{2}-4 m_{H}^{2}}}{2} t\right)
$$

where $\varphi(\mathbf{x}, t)$ does have the same time evolution described in Section 2 .

Dynamics evolves as follows. At $t=0$ a subcritical bubble is formed with initial amplitude at the core $\phi_{0}$ and radius $R$ given in ref. [8]. For $t \ll 1 / \eta$, $\left\langle\left\langle\phi^{2}(t)\right\rangle\right\rangle_{V}$ none of the modes of the initial configuration have yet relaxed to zero, even if they start to decrease as $t^{2}$. In the meanwhile, besides the noise term $\left\langle\left\langle\phi^{2}(t)\right\rangle\right\rangle_{V}^{\gamma}$ which increases as $t^{3}$, a new term begins to contribute to $\left\langle\left\langle\phi^{2}\right\rangle\right\rangle_{V}$, i.e. the term proportional to $\kappa^{2}$ increasing as $t^{2}$. For $t \gg 1 / \eta$, all the modes have relaxed towards their equilibrium value. The initial configuration has disappeared, and $\left\langle\left\langle\phi^{2}\right\rangle\right\rangle_{V}^{\text {dyn }}$ is the sum of the noise term contribution and the $\kappa^{2}$-term contribution, so that the self-consistency equation for $\left\langle\left\langle\phi^{2}\right\rangle\right\rangle_{V}^{\text {dyn }}$ reads

$$
\begin{aligned}
\left\langle\left\langle\phi^{2}\right\rangle\right\rangle_{V}^{\text {dyn }} & =f\left(\left\langle\left\langle\phi^{2}\right\rangle\right\rangle_{V}^{\text {dyn }}\right) \\
& =\kappa^{2}+\int \frac{d^{3} \mathbf{q}}{(2 \pi)^{3}} \mathrm{e}^{-|\mathbf{q}|^{2} R^{2}} \frac{T}{|\mathbf{q}|^{2}+m^{2}+3 \lambda\left\langle\left\langle\phi^{2}\right\rangle\right\rangle_{V}^{\text {dyn }}} .
\end{aligned}
$$

In Fig. 1 we present the behaviour of the function $f\left(\left\langle\left\langle\phi^{2}\right\rangle\right\rangle_{V}^{\text {dyn }}\right)$ (dashed line) in units of $1 / R_{c}$ for $\lambda=0.061, \alpha=0.048$ and $R=\xi\left(T_{f}\right)$, i.e. the variance is calculated in a typical correlation volume. Note that in the limit of very large $\left\langle\left\langle\phi^{2}\right\rangle\right\rangle_{V}^{\text {dyn }}, f$ tends toward $\phi_{-}^{2}\left(T_{f}\right) \simeq\left(24 / R_{c}\right)^{2}$.

Fig. 2 gives the same function for the two cases $R=\xi\left(T_{f}\right)$ and $R=$ $2 \xi\left(T_{f}\right)$. The points where the dashed lines intersect the solid line, representing the left hand side of eq. (55), denote the values of $\left\langle\left\langle\phi^{2}\right\rangle\right\rangle_{V}^{\text {dyn }}$ which satisfy the self-consistency equation in the two different cases: when testing the fluctuation in a correaltion volume $V_{\xi}=(4 \pi / 3) \xi^{3}$, we have

$$
\sqrt{\left\langle\left\langle\phi^{2}\right\rangle\right\rangle_{V_{\xi}}^{\text {dyn }}} \simeq\left(2.7 / R_{c}\right) \ll \phi_{-}\left(T_{f}\right) \simeq\left(24 / R_{c}\right) ;
$$

if we test the fluctuation in a volume larger than $V_{\xi}$, the variance is even smaller,

$$
\sqrt{\left\langle\left\langle\phi^{2}\right\rangle\right\rangle_{V}^{\text {dyn }}} \simeq\left(1.2 / R_{c}\right)
$$

for $R=2 \xi\left(T_{f}\right)$. Note also that our starting assumption $\eta<2 m_{H}$ is verified. 
One could naively think that the extra energy due to a nonvanishing variance would facilitate barrier penetration for the formation of critical bubbles or possibly even invalidate our thin wall approach.

We can verify that this is not the case by considering the fluctuation energy about $\phi=0$. For $R=\xi\left(T_{f}\right)$ it is given approximatly by (in units of $\left.1 / R_{c}^{4}\right)$

$$
E_{\mathrm{fl}} \simeq \frac{1}{2} m^{2}\left(T_{f}\right)\left\langle\left\langle\phi^{2}\right\rangle\right\rangle_{V}^{\mathrm{dyn}} \simeq 211 \ll-\epsilon \simeq 5 \times 10^{3},
$$

where we have made use of the fact that $\phi_{+}\left(T_{f}\right) \simeq\left(48 / R_{c}\right)$. Thus, in terms of the barrier penetration, thermal fluctuations represent only a minor correction and they do not have remarkable effect on the thin wall approximation.

From these considerations and from the fact that the variance of the thermal fluctuations $\sqrt{\left\langle\left\langle\phi^{2}\right\rangle\right\rangle_{V}^{\text {dyn }}}$ is clearly smaller than the inflection point $\phi_{-}\left(T_{f}\right)$, we can conclude that subcritical bubbles do not affect the nucleation of critical bubbles in an appreciably way.

\section{Conclusions and perspectives for future work}

In this paper we have investigated the role played by subcritical bubbles at the onset of the electroweak phase transition.

Treating the the configuration $\phi(\mathbf{x}, t)$ modelling such thermal fluctuation as a stochastic variable, we have described its dynamics by a phenomenological Langevin equation for different models. This approach allows to properly take into account both effects of the thermal bath on the system: a systematic dissipative force, which tends to erase out any initial subcritical configuration, and a random stochastic force responsible for fluctuations.

Following the evolution with time of the variance $\left\langle\left\langle\phi^{2}(t)\right\rangle\right\rangle_{V}$ in a given volume $V$, we have shown that the contribution to it from any initial subcritical configuration is quickly damped away and, in the limit of long times, $\left\langle\left\langle\phi^{2}(t)\right\rangle\right\rangle_{V}$ approaches its equilibrium value provided by the stochastic force and independent from the viscosity coefficient $\eta$, in agreement with the fluctuation-dissipation theorem.

In the most interesting case of thermal fluctuations during the electroweak phase transition, we have made use of a self-consistent Hartree approximation expected to give good results in the weak coupling limit which we believe to 
hold for a weak first order phase transition. A more correct way of proceeding might have been to make use of the variance $\left\langle\left\langle\phi^{2}(t)\right\rangle_{V}\right.$ in the substitution (50) instead of $\left\langle\left\langle\phi^{2}\right\rangle\right\rangle_{V}^{\text {dyn }}$. However, since the system reaches very quickly the equilibrium, we are confident that our approximation is reliable.

We have shown that thermal fluctuations do not affect the nucleation of critical bubbles at the tunneling temperature $T_{f}$ and from this fact we conclude that electroweak baryogenesis scenarios associated to a weak first order electroweak phase transition remain a viable possibility to explain the primordial baryon asymmetry in the Universe.

Very recently Bettencourt [9] has computed the probability at equilibrium for the fluctuations of the Standard Model Higgs field, averaged over a correlation volume, to exceed the inflection point $\phi_{-}$at $T \simeq T_{f}$ and for a physical Higgs mass $M_{H}=70 \mathrm{GeV}$, the same value we have used in our paper.

He estimated such a probability to be very small, around $1.27 \%$, and to decrease when the testing volume $V$ is increased. These estimates and behaviour with $V$ are in complete agreement with our results for the asymptotic value of $\left\langle\left\langle\phi^{2}(t)\right\rangle\right\rangle_{V}$ whose evolution with time is also given here.

The phenomenological Langevin equation used in our paper is not the true equation describing a thermal fluctuation in the hot electroweak theory.

In this respect, our work should be regarded as a first step towards a complete description of the dynamics of thermal fluctuations at the electroweak phase transition by a more complicated Langevin equation which properly describes the coupling of the stochastic field $\phi(\mathbf{x}, t)$ to the other degrees of freedom.

Nevertheless, we expect our physical results to be reliable at long times when the details on the nature of the thermal noise and the viscosity coefficient $\eta$ become unessential. On the other hand, relaxation time-scales crucially depend on the nature of the stochastic force and the strength of dissipation and their complete knowledge is needed to decide if degrees of freedom other than $\phi(\mathbf{x}, t)$ are in equilibrium or not inside the subcritical bubble.

One may realize that this is a crucial question by reminding that the effective potential (42), used to describe the free energy associated to the fluctuations, is usually obtained at 1-loop integrating out all the degrees of 
freedom of the theory other than $\phi(\mathbf{x}, t)$, i.e. fermions and gauge bosons.

In performing such a calculation, it is assumed that fermions and gauge bosons do have equilibrium distributions with a $\phi(\mathbf{x}, t)$ background dependent mass. This is true only if their interaction times with the background $\phi(\mathbf{x}, t)$ are much smaller than the typical lifetime of the subcritical bubble.

Since this condition seems not be satisfied, a full non-equilibrium approach is needed. Such an approach might lead to unexpected results as far as the issue of thermal fluctuations during the electroweak phase transition is concerned [22].

\section{Acknowledgements}

It is a pleasure to thank K. Enqvist and I. Vilja for carefully reading the manuscript and for useful suggestions. 


\section{References}

[1] For a review, see A.G. Cohen, D.B. Kaplan and A.E. Nelson, Ann. Rev. Nucl. Part. Phys. 43, 27 (1993) ; D.B. Kaplan, contribution to the $4^{\text {th }}$ International Conference on Physics Beyond the Standard Model, Lake Tahoe, 13-16 December 1994, hepph 9503360.

[2] A.D. Sakharov, Pis'ma Zh. Eksp. Fiz. 5, 32 (1967); JETP Lett. 5, 24 (1967).

[3] M. Gleiser, E.W. Kolb and R. Watkins, Nucl. Phys. B364, 411 (1991); M. Gleiser and E.W. Kolb, Phys. Rev. Lett. 69, 1304 (1992); Phys. Rev. D48, 1560 (1993); M. Gleiser and R.O. Ramos, Phys. Lett. B300, 271 (1993); N. Tetradis, Z. Phys. C57, 331 (1993).

[4] M. Dine, R. Leigh, P. Huet, A. Linde and D. Linde, Phys. Rev. D46, $550(1992)$.

[5] G. Anderson, Phys. Lett. B295, 32 (1992).

[6] G. Gelmini and M. Gleiser, Nucl. Phys. B419, 459 (1994).

[7] Particle Data Group, Review of Particle Properties, Phys. Rev. D50, 1173 (1994).

[8] K. Enqvist, A. Riotto and I. Vilja, HU-TFT/95-32 preprint, hep-ph 9505341, submitted to Phys. Rev. D.

[9] L.M.A. Bettencourt, Imperial/TP/94-95/38 preprint.

[10] M. Hindmarsh and R.J. Rivers, Nucl. Phys. B417, 506 (1994); R.J. Rivers, Fluctuations at Phase Transitions, in the Proceedings of the Nato Advanced Research Workshop on Electroweak Physics and the Early Universe, Sintra, Portugal, March 1994.

[11] See, for instance, M. Kardar, Lectures on Dynamics of Interfaces in recent Advances in Statistical physics, Istanbul Technical University, Istanbul, Turkey, July 15 to August 7, 1993.

[12] M. Kardar, G. Parisi and Y.-C. Zhang, Phys. Rev. Lett. 56, 889 (1986). 
[13] P.C. Hohenberg and B.I. Halperin, Rev. Mod. Phys. 49, 435 (1977).

[14] M. Gleiser and R.O. Ramos, Phys. Rev. D50, 2441 (1994); B.L. Hu, J.P. Paz and Y. Zhang, in The Origin of Structure in the Universe, Ed. E. Gunzig and P. Nardone (Kluwer Acad. Publ. 1993); D. Lee and D. Boyanowsky, Nucl. Phys. B406, 631 (1993); S. Habib, in Stochastic Processes in Astrophysics, Proc. Eigth Annual Workshop in Nonlinear Astronomy (1993),

[15] G.H. Derrick, J. Math. Phys. 5, 1252 (1964) .

[16] See also E.J. Copeland, M. Gleiser and H.-R. Muller, Fermilab-Pub95/021-A preprint.

[17] S.K. Ma, Rev. Mod. Phys. 45, 589 (1973).

[18] K. Kajantie, K. Rummukainen and M. Shaposnikov, Nucl. Phys. B407, 27 (1993); K. Farakos, K. Kajantie, K. Rummukainen and M.E. Shaposhnikov, Phys. Lett. B336, 494 (1994); B. Bunk, E.M. Ilgenfriz, J. Kripfganz and A. Schiller, Nucl. Phys. B403, 453 (1993); F. Csikor et al., Phys. Lett. B334, 405 (1994).

[19] Z. Fodor and Hebecker, Nucl. Phys. B432, 127 (1994).

[20] K. Enqvist, J. Ignatius, K. Kajantie and K. Rummukainen, Phys. Rev. D45, 3415 (1992).

[21] P. Elmfors, K. Enqvist and I. Vilja, Nucl. Phys. B412, 459 (1994).

[22] I. Vilja and A. Riotto, in preparation. 


\section{Figure Captions}

Figure 1: The plot of the function $f\left(\left\langle\left\langle\phi^{2}\right\rangle\right\rangle_{V}^{\text {dyn }}\right)$ (dashed line) in units of $1 / R_{c}^{2}$ for $\lambda=0.061, \alpha=0.048$ and $R=\xi\left(T_{f}\right)$. The solid line represents the inflection point $\phi_{-}^{2}\left(T_{f}\right)$.

Figure 1: The plot of the function $f\left(\left\langle\left\langle\phi^{2}\right\rangle\right\rangle_{V}^{\text {dyn }}\right)$ (dashed lines) in units of $1 / R_{c}^{2}$ for the same values of $\lambda$ and $\alpha$ given in Figure 1 and for $R=\xi\left(T_{f}\right)$ and $R=2 \xi\left(T_{f}\right)$ respectively. The intersection points between the dashed lines and the solid line represent the values of $\left\langle\left\langle\phi^{2}\right\rangle\right\rangle_{V}^{\text {dyn }}$ satisfying the selfconsistency equation in the two cases, see the text. 


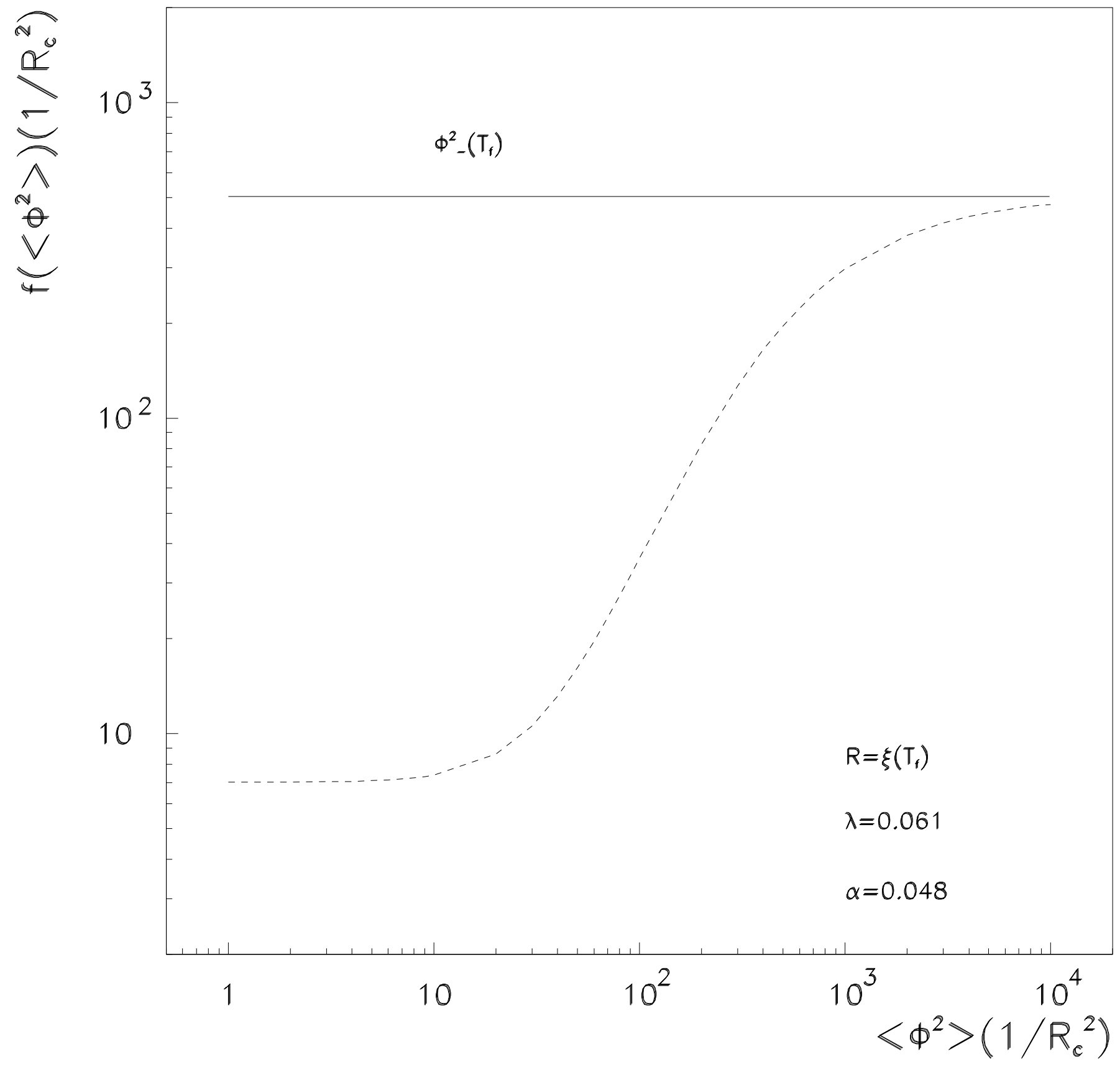




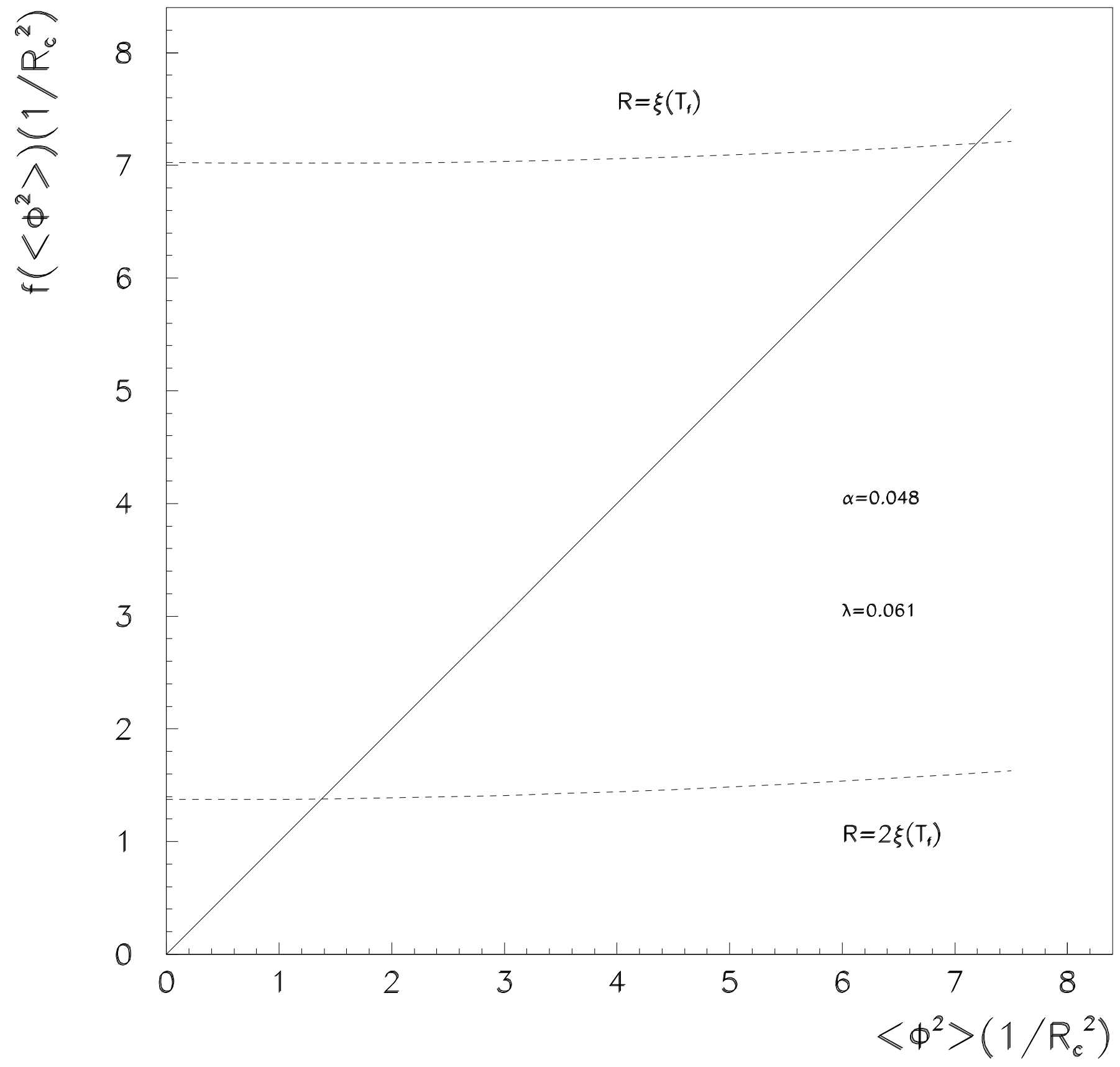

